\title{
GENERATORS FOR ALGEBRAS OF RELATIONS ${ }^{1}$
}

\author{
BY A. R. BEDNAREK AND S. M. ULAM
}

Communicated by J. T. Schwartz, May 11, 1976

Let $B_{n}$ denote the collection of all binary relations on the set $X=\{1,2$, $\ldots, n\}$. The purpose of this paper is to observe that there exists a pair of relations on $X$ that generate all of $B_{n}$ under the boolean operations and relational composition.

In [1] C. J. Everett and S. M. Ulam introduced the notion of an abstract projective algebra. McKinsey [2] showed that every projective algebra is isomorphic to a subalgebra of a complete atomic projective algebra and thus, in view of the representation given in [1], every projective algebra is isomorphic to a projective algebra of subsets of a direct product; that is, to an algebra of relations.

Projective Algebra. A boolean algebra $P$ with unit 1 and zero 0 , so that for all $x \in P, 0 \leqslant x \leqslant 1$, is said to be a projective algebra if there are defined two mappings $\pi_{1}$ and $\pi_{2}$ of $P$ into $P$ satisfying the following:

$\mathrm{P}_{1} \cdot \pi_{i}(a \vee b)=\pi_{i} a \vee \pi_{i} b$.

$\mathrm{P}_{2} . \pi_{1} \pi_{2} 1=p_{0}=\pi_{2} \pi_{1} 1$ where $p_{0}$ is an atom of $P$.

$\mathrm{P}_{3}, \pi_{i} a=0$ if and only if $a=0$.

$\mathrm{P}_{4}, \pi_{i} \pi_{i} a=\pi_{i} a$.

$\mathrm{P}_{5}$. For $0<a \leqslant \pi_{1} 1,0<b \leqslant \pi_{2} 1$, there exists an element $a \square b$ such that $\pi_{1}(a \square b)=a, \pi_{2}(a \square b)=b$, with the property that $x \in P, \pi_{1} x=a, \pi_{2} x=$ $b$ implies $x \leqslant a \square b$.

$\mathrm{P}_{6} \cdot \pi_{1} 1 \square p_{0}=\pi_{1} 1 ; p_{0} \square \pi_{2} 1=\pi_{2} 1$.

$\mathrm{P}_{7} .0<x, y \leqslant \pi_{1} 1$ implies $(x \vee y) \square \pi_{2} 1=\left(x \square \pi_{2} 1\right) \vee\left(y \square \pi_{2} 1\right)$; and $0<u, v \leqslant \pi_{2} 1$ implies $\pi_{1} 1 \square(u \vee v)=\left(\pi_{1} 1 \square u\right) \vee\left(\pi_{1} 1 \square v\right)$.

If the projective algebra $P$ is a complete atomic boolean algebra, then $P$ is called a complete atomic projective algebra. The projective algebra $P$ is said to be projectively generated by a subset $A$ if $P$ can be obtained from $A$ using $\pi_{1}, \pi_{2}$, $\square$ and the boolean operations.

Consider $B_{n}$ and let $p_{0}=(1,1)$. We define the mappings $\pi_{1}, \pi_{2}: B_{n} \rightarrow$ $B_{n}$ and a product $\square: B_{n} \times B_{n} \rightarrow B_{n}$ as follows:

(i) $\pi_{1} \alpha=\alpha\left((X \times X) p_{0}\right)$,

(ii) $\pi_{2} \alpha=\left(p_{0}(X \times X)\right) \alpha$,

(iii) $\alpha \square \beta=(\alpha(X \times X)) \beta$,

AMS (MOS) subject classifications (1970). Primary $02 \mathrm{~J} 10$.

${ }^{1}$ Research supported by NSF Grant No. MSC75-21130. 
where juxtaposition denotes the composition of relations.

It is easy to verify axioms $P_{1}-P_{7}$ to establish that $B_{n}$ with the atom $p_{0}$ and the mappings $\pi_{1}, \pi_{2}$ and $\square$ as defined is a projective algebra.

The verification above, as well as the calculations below are made easier by noting the following equivalent forms of (i), (ii) and (iii):

(i) $\pi_{1} \alpha=$ domain $(\alpha) \times\{1\}$;

(ii) $\pi_{2} \alpha=\{1\} \times$ range $(\alpha)$;

(iii) $\alpha \square \beta=\operatorname{domain}(\alpha) \times \operatorname{range}(\beta)$.

THEOREM 1. The projective algebra $B_{n}$ can be projectively generated by a pair of disjoint elements.

Proof. We observe first that if we generate the atoms $(1, k)$ and $(k, 1), 1$ $\leqslant k \leqslant n$, then all others are obtained by taking the $\square$-product of suitable pairs of these.

Let $\alpha_{0}=\{(x, y) \mid x<y\}$ and $\beta_{0}=\{(x, y) \mid y<x\}$. Now $p_{0}=(1,1)=$ $\pi_{2} \pi_{1} \beta_{0}$. If we let $\alpha_{1}=\alpha_{0}-\left(p_{0} \square \pi_{2} \alpha_{0}\right)$ and $\beta_{1}=\beta_{0}-\left(\pi_{1} \beta_{0} \square p_{0}\right)$, we get $\left(\pi_{1} \alpha_{1}-\pi_{1} \beta_{1}\right)=(2,1)$ and $\left(\pi_{2} \beta_{1}-\pi_{2} \alpha_{1}\right)=(1,2)$. Using the recursions $\alpha_{k+1}$ $=\alpha_{k}-\left((k+1,1) \square \pi_{2} \alpha_{k}\right)$ and $\beta_{k+1}=\beta_{k}-\left(\pi_{1} \beta_{k} \square(1, k+1)\right)$, noting that $\alpha_{k}=\{(x, y) \mid k<x<y\}$ and $\beta_{k}=\{(x, y) \mid k<y<x\}$, we see that $\left(\pi_{1} \alpha_{k}-\right.$ $\left.\pi_{1} \beta_{k}\right)=(k+1,1)$ and $\left(\pi_{2} \beta_{k}-\pi_{2} \alpha_{k}\right)=(1, k+1)$, for all $0 \leqslant k \leqslant n-2$. Also $\pi_{1} \beta_{n-2}=(n, 1)$ and $\pi_{2} \alpha_{n-2}=(1, n)$, so that we have generated all of the atoms mentioned above.

THEOREM 2. The algebra of relations $B_{n}$ can be generated, with respect to the boolean operations and composition, by two relations.

Proof. Let $\bar{\alpha}=\alpha_{0} \cup\{(1,1)\}$ and $\bar{\beta}=\beta_{0} \cup\{(1,1)\}$. Now $\bar{\alpha} \cap \bar{\beta}=$ $\{(1,1)\}=p_{0}, \bar{\alpha} \cup \bar{\beta} \cup \bar{\beta} \bar{\alpha}=X \times X, \alpha_{0}=\bar{\alpha}-p_{0}$ and $\beta_{0}=\bar{\beta}-p_{0}$. Since we defined the mappings $\pi_{1}, \pi_{2}$ and $\square$ in terms of the composition in $B_{n}$, Theorem 2 is an immediate consequence of Theorem 1 .

REMARK. It is well-known that $B_{n}$ cannot be generated by a pair of elements using only the boolean operations. Moreover one can show that the compositional semigroup $B_{n}$ cannot be generated by a pair of relations.

\section{REFERENCES}

1. C. J. Everett, Jr., and S. M. Ulam, Projective algebra. I, Amer. J. Math. 68 (1946), 77-88. MR 7, 409.

2. J. C. C. McKinsey, On the representation of projective algebras, Amer. J. Math. 70 (1948), 375-384. MR 10, 4.

DEPARTMENT OF MATHEMATICS, UNIVERSITY OF FLORIDA, GAINESVILLE, FLORIDA 32611

DEPARTMENT OF MATHEMATICS, UNIVERSITY OF COLORADO, BOULDER, COLORADO 80302 